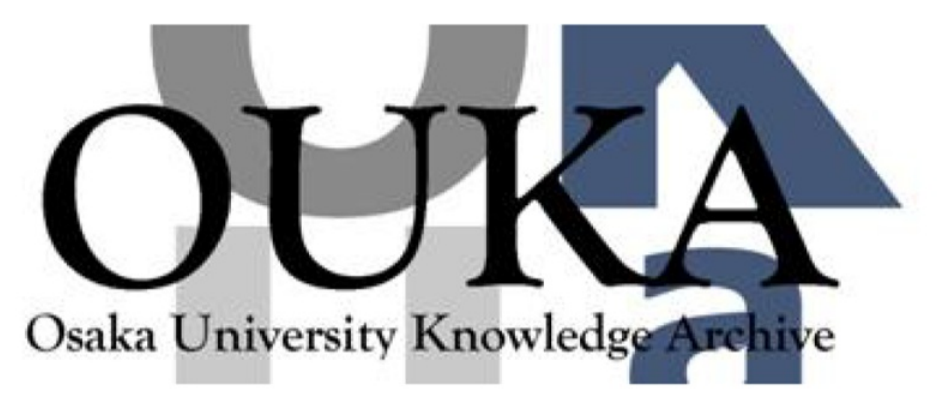

\begin{tabular}{|c|l|}
\hline Title & $\begin{array}{l}\text { Observation of a stacking process of } \\
\text { microparticles with multiple beams }\end{array}$ \\
\hline Author(s) & Sumiyama, Fumika; Ogura, Yusuke; Tanida, Jun \\
\hline Citation & Applied Optics. 44(16) p. 3271-p.3275 \\
\hline Issue Date & $2005-06-01$ \\
\hline oaire:version & VoR \\
\hline URL & https://hdl. handle.net/11094/3232 \\
\hline rights & \\
\hline Note & \\
\hline
\end{tabular}

Osaka University Knowledge Archive : OUKA

https://ir. Library. osaka-u. ac. jp/

Osaka University 


\title{
Observation of a stacking process of microparticles with multiple beams
}

\author{
Fumika Sumiyama, Yusuke Ogura, and Jun Tanida
}

\begin{abstract}
We developed a method of constructing a stacked structure of microparticles by using multiple beams emitted by a vertical-cavity surface-emitting laser array [Appl. Phys. Lett. 82, 2969 (2003)]. We construct a new observation system to observe the stacked structure and to clarify the stacking process. Results confirmed that the stacked structure was lifted up from the sample stage and fabricated without space between the microparticles. Furthermore, we investigated the difference of stacking processes when 2 $\times 2$ beams and a single beam were used. As a result, we found that a stacked structure, when $2 \times 2$ beams were used, can be fabricated with lower optical power than when a single beam was used. (C) 2005 Optical Society of America
\end{abstract}

OCIS codes: $140.7010,250.7260,170.4520,220.4610$.

\section{Introduction}

We previously demonstrated that an array of verticalcavity surface-emitting lasers (VCSELs), as a new type of equipment for optical manipulation, provides attractive features. ${ }^{1,2}$ Controllability of the operation and compactness of the required optical system are notable features of optical manipulation with the VCSEL array. With the technique, multiple small particles can be translated without any mechanical movement. In addition, we have found that four beams arranged in a square pattern can build up a stacked structure of microparticles. ${ }^{3}$ One of the advantages of the stacking is that a number of microparticles can be translated with a few beams. Usually, one beam was used for translation of one microparticle, so when multiple microparticles were translated simultaneously, we often needed the same number of beams as target microparticles. By using our method, we can translate multiple microparticles with a simple operation and utilize the light power efficiently. We can fabricate two- and three-dimensional structures of microparticles by using this method, so two- and three-dimensional struc-

The authors are with the Department of Information and Physical Sciences, Graduate School of Information, Science, and Technology, Osaka University, 2-1 Yamadaoka, Suita, Osaka 565-0871, Japan. The e-mail address for F. Sumiyama is sumiyama@ ist.osaka-u.ac.jp

Received 3 September 2004; revised manuscript received 13 January 2005; accepted 14 January 2005.

$0003-6935 / 05 / 163271-05 \$ 15.00 / 0$

(C) 2005 Optical Society of America ture fabrications of a photonic crystal are considered one application. In addition, the microparticles were fabricated in a stacked structure without threedimensional beam scanning. So, we can do threedimensional dynamic operation of microparticles with a simple operation. It is expected that this method can be applied to micromixers and micropumps in a labon-a-chip-based system. Although this phenomenon is interesting and has potential applications, fine details of the stacking process were not revealed owing to the restricted view angle during observation. In this study we construct a new observation system in which the side view of the stacking process can be captured, and this process is clarified.

Optical manipulation is a technique for handling a small object by use of interaction between light and the object. Since Ashkin et al. demonstrated the technique of three-dimensional micro-object manipulation by using the gradient force of light in $1986,{ }^{4}$ this technique has been utilized as a versatile tool for translating objects without physical contact or distraction. Optical manipulation is particularly effective for biological applications including cell manipulation ${ }^{5}$ and measurement of molecular functionalities.6,7 Furthermore, fabrication of three-dimensional structures was recently demonstrated by use of beams propagated along the optical axis. For example, a three-dimensional structure was created by the intensity distribution of four beams generated by interference of two LaguerreGaussian light beams, ${ }^{8}$ and simultaneous micromanipulation in multiple planes was demonstrated with the nondiffractive property of a Bessel beam. ${ }^{9}$ These results show that control of emission patterns is an 
effective method for extending the application fields of optical manipulation. The typical equipment for optical manipulation consists of a high-power light source such as a YAG laser and an external beam-control device, e.g., a beam scanner or a spatial light modulator. ${ }^{10,11}$ The fundamentals of the equipment seem well established, but the features provided by the VCSEL device are expected to extend applicable fields of this state-of-the-art technology.

In this paper we report on experimental results of microparticle stacking by using a VCSEL array source with side-view observation. First, we explain the VCSEL trapping system, including modifications for side-view observation. We then present the observed phenomena of the stacking process. The difference between the cases of $2 \times 2$ beams and a single beam is clarified by means of comparing the stacking processes. Finally, we comment on the features of optical trapping with the VCSEL array.

\section{Vertical-Cavity Surface-Emitting Laser Array Trapping System}

We have developed a new type of optical manipulation system by using a VCSEL array. In this system we irradiate multiple beams to the sample stage from below and flexibly manipulate microparticles. We use a VCSEL array device (854-nm wavelength, $8 \times 8$ pixels, $250-\mu \mathrm{m}$ pixel pitch, $15-\mu \mathrm{m}$ aperture, and 5.1-mW maximum emission power) provided by the NTT (Nippon Telegraph and Telephone Corporation) Photonics Laboratory. The emission distribution of the VCSEL array is controlled with a personal computer (Dell, 800-MHz Pentium III processor) through a digital-to-analog converter and VCSEL driver circuits. Each of the VCSELs is driven by a $500-\mathrm{Hz}$ rectangular wave signal to prevent the device from overloading. We set a microlens array in front of the VCSEL to reduce the loss of beam power. The focal length and the pitch of the microlens array are 720 and $250 \mu \mathrm{m}$, respectively. We use a waterimmersion, long-working-distance objective lens (Olympus LUMPlan, $60 \times, 0.90$ N.A.) as the focusing lens. The beams are constantly focused at the plane that is $20 \mu \mathrm{m}$ above the sample stage because we could stack the maximum number of microparticles with this experimental condition. The pixels of the VCSEL array are imaged onto the focal plane with a magnification of $1 / 67$, so that the optical spot pitch on the focal plane is $3.75 \mu \mathrm{m}$. The average light power is $1.0 \mathrm{~mW}$, and the diameter of the spot is $2.6 \mu \mathrm{m}$.

Previously, we observed the microparticles with a water-immersion, long-working-distance objective lens (Olympus LUMPlan, $100 \times, 1.0$ N.A.) from the top with a CCD. This method, however, made it difficult to capture the motion of the microparticles along the optical axis because we observed the sample from the top only. As a solution, we have improved the system for side-on observation of the microparticle-stacking process. Figure 1 illustrates the experimental setup of the modified VCSEL array manipulation system. The major modification to the system is the addition of an objective lens for side-

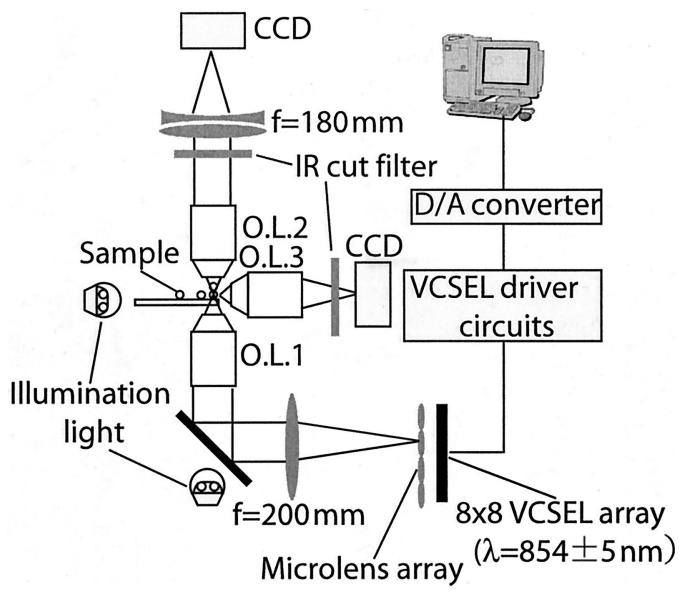

Fig. 1. Experimental setup. O.L. 1 and O.L. 2, water-immersible long-distance objective lens; O.L. 3, super-long-distance objective lens; D/A, digital-to-analog.

view observation, allowing us to observe the stacked structure from the top and the side simultaneously. In this system we use a super-long-working-distance objective lens (Mitsutoyo, MPlan Apo SL 50×, 0.42 N.A., working distance $20.5 \mathrm{~mm}$ ) to avoid interference with the objective lens for top-view observation.

In our experiments the sample objects were polystyrene particles (Polysciences, Inc., Polybead polystyrene microspheres) with a 1.60 refractive index. We dispersed the 10- $\mu$ m-diameter microparticles on a glass slide that had a shallow hole in order to make sufficient space to fabricate the stacked structure. Because of the limitations of the optical setup, the glass slide was cut at the center so that the working space made by the hole and the cover glass was located at the edge. We fabricated a stacked structure near the edge and observed the process from the side.

\section{Experimental Result of Stacking Microparticles}

In the experiment we fabricated a stacked structure by translating the sample stage horizontally to capture a new microparticle. Under the previous experimental system, we observed the stacking process only from the top, which made the stacked microparticles appear to overlap at the same position. As a result, when more microparticles were stacked, it was difficult to observe all the microparticles in the stacked structure simultaneously because the top and the bottom were relatively displaced beyond the focusing range. Therefore, only after the VCSELs were turned off and the microparticles fell down onto the sample stage could we confirm the existence of the microparticles that were not observed.

In our improved system, we can clearly confirm the condition of microparticles in the stacked structure by side-view observation of the structure. Figure 2 shows a result of side-view observation of the stacking process. At first, a microparticle illuminated from the bottom was lifted up at the average speed of $2 \mu \mathrm{m} / \mathrm{s}$ [Fig. 2(a)]. The target microparticle stopped rising at $39 \mu \mathrm{m}$ above the sample stage [Fig. 2(b)]. 


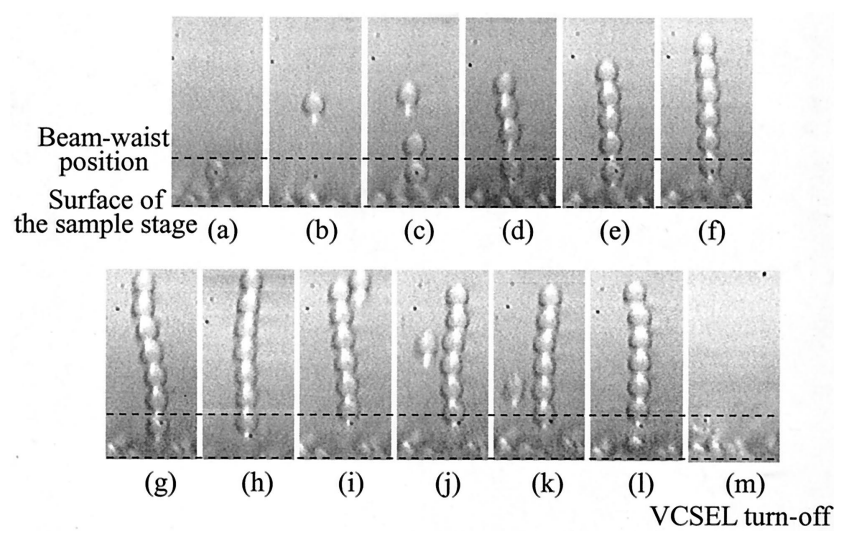

Fig. 2. Side-view images of the stacking process. (We captured images at intervals of $15 \mathrm{~s}$.)

Second, we moved the sample stage to illuminate another microparticle. The previously captured microparticle maintained its horizontal and vertical position owing to light pressure. When the newly captured microparticle began to be lifted up immediately after being illuminated, the previously captured microparticle also began to rise [Fig. 2(c)]. The position at which the previously captured microparticle stopped rising depended on the number of the newly captured microparticles. It is thought that the newly captured microparticles influenced the irradiation pattern to the previously captured microparticle, so the position at which the levitation force balanced with the gravity was changed. When we moved the sample stage again, the microparticles maintained their positions. This result indicates that we could translate the stacked particles in relation to the sample stage. Repeating the same procedure, we could lift up multiple microparticles and fabricate a stacked structure. We could stack seven microparticles under the present experimental condition [Fig. 2(h)].

From the experimental result, it was found that the stacked structure was lifted above the sample stage and fabricated without space between the microparticles. After the maximum number of microparticles was stacked, the top microparticle would fall off the stack when another microparticle was added [Fig. 2(i)]. The reason is considered to be that the light pressure applied to the top microparticle of the stacked structure becomes too weak to maintain a stable structure because the VCSEL beams were re-

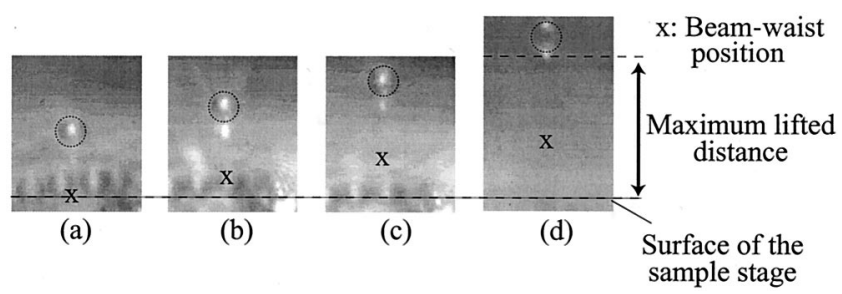

Fig. 3. Side-view image of the lifted microparticles when the distance between the beam waist and the sample stage was (a) 0 , (b) 10 , (c) 20 , (d) $30 \mu \mathrm{m}$.
Table 1. Variation of Maximum Lifted Distance and Maximum Number of Stacked Microparticles for the Change of the Focal Point

\begin{tabular}{ccc}
\hline $\begin{array}{c}\text { Distance between the } \\
\begin{array}{c}\text { Focal Spot and the } \\
\text { Sample Stage }(\mu \mathrm{m})\end{array}\end{array}$ & $\begin{array}{c}\text { Maximum Lifted } \\
\text { Distance }(\mu \mathrm{m})\end{array}$ & $\begin{array}{c}\text { Maximum Number } \\
\text { of Stacked } \\
\text { Microparticles }\end{array}$ \\
\hline 0 & 27 & 2 \\
10 & 39 & 5 \\
20 & 60 & 7 \\
30 & 72 & 6 \\
\hline
\end{tabular}

duced owing to scattering and absorption by the lower microparticles. Additionally, we observed an interesting phenomenon that the microparticle that fell down from the top position could be captured again without one's moving the sample stage [Figs. 2(i)-2(1)]. This suggests that we can circulate the microparticles in the stacked structure. In our experiment we could circulate the microparticles four times. The speed of circulation was approximately $60 \mathrm{~s} /$ circle. It is expected that this method can be used as a micropump or a micromixer in a lab-on-achip-based system.

We have confirmed that the beam waist position affects the maximum number of stacked microparticles. $^{3}$ In the experiment we investigated the effect of the beam waist position on the motion of microparticles in the stacked structure by observing from the side. Figure 3 and Table 1 show the side view of the lifted microparticle when the distance between the beam waist and the sample stage was $0,10,20$, and $30 \mu \mathrm{m}$. This result indicates that raising the beam waist position increases the maximum lifted distance of microparticles. However, when the distance was $30 \mu \mathrm{m}$, the maximum number of stacked microparticles was reduced. The reason is considered to be that the levitation force was changed owing to the distance between the beam waist and the sample stage.

Finally, we show a model considered to represent the stacking process. Figure 4 shows the model of the microparticle-stacking process. We use $2 \times 2$ pixels of the VCSEL array and focus the beams around a microparticle. In our experiment the size of the optical

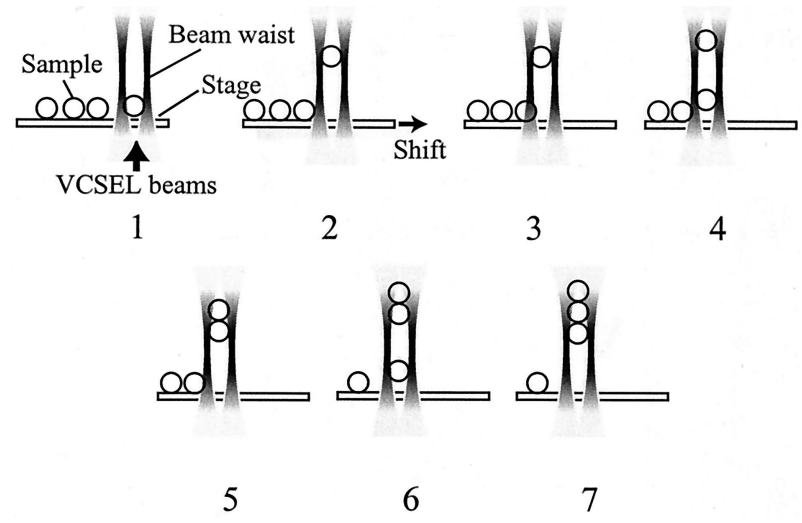

Fig. 4. Model of the microparticle-stacking process. 


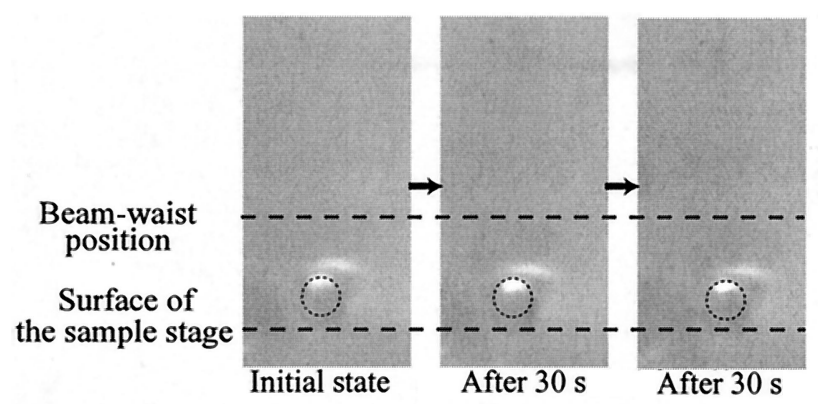

(a)

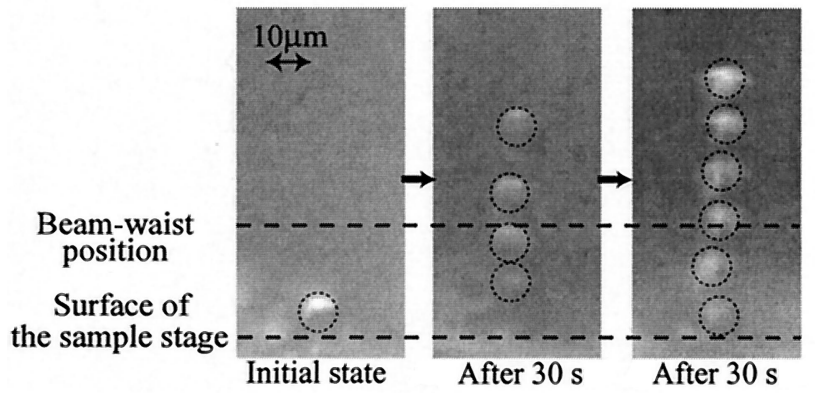

(b)

Fig. 5. Side view of the stacked microparticle structure fabricated with a single beam (a) when we do not move the sample stage and (b) when we keep moving the sample stage.

field illuminated by $2 \times 2$ beams on the sample stage was $15.5 \mu \mathrm{m}$. Therefore the $10-\mu \mathrm{m}$-diameter microparticles were confined in the optical field. The new microparticle is then lifted by the light pressure. Following that, we move the sample stage to focus the beams around another microparticle. As soon as a newly captured microparticle begins to be lifted up, the previously captured microparticle also begins to rise. The second microparticle is lifted up to the position at which the previously captured microparticle stopped rising. Repeating this procedure, we can stack microparticles along the propagating direction of $2 \times 2$ beams. The reason is that the stacked structure is formed from the microparticle lifted first toward the down direction.

Balance of the levitation force and gravity is also important to fabricate a stable structure. This condition is dependent on the irradiation pattern and the optical intensity of the trap beams. In Section 4 we show the effect of the irradiation pattern on a stacked structure fabrication.

\section{Comparison of $2 \times 2$ Beams and a Single Beam Trapping}

We attempted to achieve stacking microparticles with a single beam to compare it with the case of 2 $\times 2$ beams. We used a He-Ne laser (632.8-nm wavelength) as the light source of a single beam and fabricated a stacked structure with the same procedure as the case of $2 \times 2$ beams. The light power of the $\mathrm{He}-\mathrm{Ne}$ laser was $4.0 \mathrm{~mW}$, which is the same as the total optical power of the $2 \times 2$ beams, and the diameter of the spot was $2.7 \mu \mathrm{m}$. Figure 5 shows a side view of the stacking process with a single beam. As can be seen from the figure, when we did not move the sample stage, we could not lift up the microparticles [Fig. 5(a)]. In contrast, by moving the sample stage at approximately $10 \mu \mathrm{m} / \mathrm{s}$, we successfully lifted the initial microparticle and fabricated the stacked structure [Fig. 5(b)]. Consequently, delicate manipulation of the sample stage is required to form a stacked structure with a single beam. When we use more than a $10-\mathrm{mW}$ single beam, we can lift microparticles and fabricate the stacked structure without moving the sample stage.

The levitation force on a microparticle is dependent on the illuminated position of the microparticle. A numerical calculation predicted that the levitation force induced by illumination of the edge of a microparticle is stronger than that by illumination of the center. ${ }^{2}$ In the experiment, when a single beam is used, the microparticle was captured at the center of the beam, and, as long as we did not move the sample stage, the single beam always illuminated the center of the microparticle. Under our experimental condition (optical power: $1.0 \mathrm{~mW}$ ), we could not lift up the microparticle because the microparticle could not receive enough levitation force. In contrast, when we moved the sample stage, we could lift up the microparticles with a single beam of the same optical power because we could shift the illuminated position to the edge of the microparticle and raise the levitation force. As a result, the microparticles could be lifted up with a single beam, and a stacked structure was fabricated. However, the fabricated stacked structure was easily broken in the unstable condition because the sample stage was kept moving in order to lift up the microparticles. When we used $2 \times 2$ beams, the microparticles could be lifted up efficiently even if we did not move the sample stage because the microparticle was captured at the center of $2 \times 2$ beams and the edge of the microparticles were always illuminated by the beam. Therefore the microparticles could receive a sufficient levitation force to be lifted.

The illumination pattern is an important factor for fabricating a stacked structure. When we measured the power required for stacking seven microparticles, $4.0 \mathrm{~mW}$ of power was needed with $2 \times 2$ beams, whereas $6.0 \mathrm{~mW}$ was required with a single beam. Especially when we used a single beam, the top microparticle could not follow the move of the stage and fell down; thus we needed stronger optical power to prevent the microparticle from falling down. We confirmed that the comparison of the condition of stacked structures shows the stacked structure fabricated with $2 \times 2$ beams is more stable than that fabricated with a single beam. In particular, when we used a single beam, the distance of the microparticles kept changing while we fabricated a stacked structure. It is thought that the path and condition of a beam that propagates in the microparticles influenced the stacked structure. We conclude that the use of 2 $\times 2$ beams is more effective than the use of a single beam for fabricating a stacked structure. 


\section{Conclusion}

A notable achievement in this study is that we clarified a fabricating process for a stacked structure by using VCSEL array trapping by observing from the side and the top simultaneously. Results confirmed that the stacked structure was lifted up from the sample stage and fabricated without space between the microparticles. We also compared the stacking process performed with a single beam with that by $2 \times 2$ VCSEL beams. In the experiment we found that the stacked structure can be fabricated with lower optical power than when a single beam is used. The reason seems to be that $2 \times 2$ beams could lift up the microparticles with a weaker optical power beam without beam scanning because the edge of the microparticles was irradiated. This is a notable feature of this stacking method with $2 \times 2$ VCSEL beams. The $2 \times 2$ beam illumination is particularly effective for stacking cells or other biological samples without causing damage by heat. Furthermore, micromixers and micropumps in the lab on a chip and threedimensional structure fabrication of the photonic crystal are considered an application of this method.

This research is partially supported by Ministry of Education, Science, Sports, and Culture, Grant-inAid for Scientific Research (A), 15200023, 2003, and the Core Research for Evolutional Science and Technology of the Japan Science and Technology Agency.

\section{References}

1. Y. Ogura, K. Kagawa, and J. Tanida, "Optical manipulation of microscopic objects by means of vertical-cavity surface- emitting laser array sources," Appl. Opt. 40, 5430-5453 (2001).

2. Y. Ogura, N. Shirai, and J. Tanida, "Optical levitation and translation of a microscopic particle by use of multiple beams generated by vertical-cavity surface-emitting laser array sources," Appl. Opt. 41, 5645-5654 (2002).

3. F. Sumiyama, Y. Ogura, and J. Tanida, "Stacking and translation of microscopic particles by means of $2 \times 2$ beams emitted from a vertical-cavity surface-emitting laser array," Appl. Phys. Lett. 82, 2969-2971 (2003).

4. A. Ashkin, J. M. Dziedzic, J. E. Bjorkholm, and S. Chu, "Observation of a single-beam gradient force optical trap for dielectric particles," Opt. Lett. 11, 288-290 (1986).

5. S. C. Grover, A. G. Skirtach, R. C. Gauthier, and C. P. Grover, "Automated single-cell sorting system based on optical trapping," J. Biomed. Opt. 6, 14-22 (2001).

6. Z. Bryant, M. D. Stone, J. Gore, S. B. Smith, N. R. Cozzarelli, and C. Bustamante, "Structural transition and elasticity from torque measurements on DNA," Nature 424, 338-341 (2003).

7. H. Tanaka, K. Homma, A. H. Iwane, E. Katayama, R. Ikebe, J. Saito, T. Yanagida, and M. Ikebe, "The motor domain determines the large step of myosin-V," Nature 415, 192-195 (2002).

8. M. P. MacDonald, L. Paterson, K. Volke-Sepulveda, J. Arlt, W. Sibbett, and K. Dholakia, "Creation and manipulation of threedimensional optically trapped structures," Science 296, 11011103 (2002).

9. V. G. Chavez, D. McGloin, H. Melville, W. Sibbet, and K. Dholakia, "Simultaneous micromanipulation in multiple planes using a self-reconstructing light beam," Nature 419, 145-147 (2002).

10. C. Mio, T. Gong, A. Terray, and D. W. M. Marr, "Design of a scanning laser optical trap for multiparticle manipulation," Rev. Sci. Instrum. 71, 2196-2200 (2000).

11. J. E. Curtis, B. A. Koss, and D. Grier, "Dynamic holographic optical tweezers," Opt. Commun. 207, 169-175 (2002). 